\title{
Analysis and design of gain-scheduling blade-pitch controllers for wind turbine down- regulation*
}

Lio, Alan Wai Hou; Galinos, Christos; Meseguer Urban, Albert

Published in:

Proceedings of the 15th International Conference on Control and Automation (ICCA)

Link to article, DOI:

10.1109/ICCA.2019.8899611

Publication date:

2019

Document Version

Peer reviewed version

Link back to DTU Orbit

Citation (APA):

Lio, A. W. H., Galinos, C., \& Meseguer Urban, A. (2019). Analysis and design of gain-scheduling blade-pitch controllers for wind turbine down-regulation*. In Proceedings of the 15th International Conference on Control and Automation (ICCA) (pp. 708-712). IEEE. IEEE International Conference on Control and Automation https://doi.org/10.1109/ICCA.2019.8899611

\section{General rights}

Copyright and moral rights for the publications made accessible in the public portal are retained by the authors and/or other copyright owners and it is a condition of accessing publications that users recognise and abide by the legal requirements associated with these rights.

- Users may download and print one copy of any publication from the public portal for the purpose of private study or research.

- You may not further distribute the material or use it for any profit-making activity or commercial gain

- You may freely distribute the URL identifying the publication in the public portal 


\title{
Analysis and design of gain-scheduling blade-pitch controllers for wind turbine down-regulation*
}

\author{
Wai Hou Lio ${ }^{1,2}$, Christos Galinos ${ }^{1}$ and Albert Meseguer Urbán ${ }^{1}$
}

\begin{abstract}
The use of down-regulation control strategies on turbines offers a means of stabilising the grid or improving the efficiency of the wind farm. Nonetheless, these de-rating strategies are often imposed upon an existing blade-pitch control structure, resulting in sub-optimal or poor performance. In particular, such an approach would potentially deteriorate the robustness of the rotor speed control loop and excite other turbine dynamics. Thus, this work proposes a blade-pitch control design for de-rating operation, where the strategy takes into account the changes in the aerodynamic characteristics of the turbine caused by down-regulation. Most importantly, the proposed controller retained the desired control bandwidth and robustness properties in both nominal and de-rating operations. Numerical simulations showed that an improvement on the pitch activities during down-regulation could lead to better reductions in the fatigue loads of some key turbine components.
\end{abstract}

\section{INTRODUCTION}

Large wind turbines are often curtailed or down-regulated for the purposes of improving the wind farm efficiency or stabilising the grid. One of the examples is that a reduction in the power/thrust of the upstream turbine could improve the aerodynamic wake situation for the downstream turbines [1], [2]. In the literature, there exist numerous de-rating strategies characterised by the rotor speed set-point for down-regulating a turbine [3]-[5]. One strategy $(\operatorname{Max}-\Omega)$ is that for a given de-rated power demand, the rotor speed is maintained at a rate set by the blade-pitch controller and the generator torque decreases accordingly, whereas another strategy (Const- $\Omega$ ) reduces the rotor speed for achieving the de-rated power output. Both down-regulation methods require the use of the blade-pitch controller to track the rotor speed set-point. Nonetheless, the blade-pitch control design for nominal or de-rating operations often remains the same or overlooked.

In nominal operations, the blade-pitch controller ensures the rotor speed to track the rated value, typically, via proportional-integral (PI) design (e.g. [6]). The blade-pitch is adjusted in response to the rotor speed variations and such variations are mainly caused by the wind-induced aerodynamic torque. The aerodynamic torque on the turbine blade is well-known as a non-linear function of the bladepitch angle, rotor speed and wind speed. Thus, the bladepitch controller often adopted a gain-scheduling approach based on the turbine operating conditions for improving the pitch controller performance and robustness (e.g. [6]-[8]).

*This research was supported by the PowerKey project (EUDP Project No. 12558).

${ }^{1}$ Department of Wind Energy, Technical University of Denmark, DK4000 Roskilde, Denmark.

2 Corresponding author. wali@dtu.dk
In down-regulation, the operating conditions of the turbine are different from those in nominal operations. The majority of wind turbine down-regulation studies often simply imposed the de-rating strategies upon the existing bladepitch control structure, that could potentially lead to a suboptimal performance or excitation of other turbine dynamics. Consequently, this work aims to address this problem by investigating the gain-scheduling blade-pitch controller during down-regulation. The contributions of this work are twofold.

- Firstly, a counterexample demonstrates the adverse effects on the system robustness and performance when the de-rating strategy is simply imposed on an existing pitch control structure.

- Secondly, a new method is proposed to retain the robustness and performance of the blade-pitch control loop during down-regulation.

The remainder of this paper is structured as follows. In Section II, the background on the nominal blade-pitch controller is presented. It is followed in Section III by the motivating example and proposed design for the bladepitch controller in down-regulation. In Section IV, simulation results on the high-fidelity turbine demonstrate the performance of the baseline and proposed controller in downregulation. Conclusions are in Section V.

\section{DESIGN OF THE NOMINAL BLADE-PITCH CONTROLLER}

This section presents the nominal pitch controller design. Considering the simplified non-linear drive-train dynamics of the turbine, assuming the shaft is rigid, defined as follows (e.g. [6]):

$$
J_{\mathrm{r}} \dot{\Omega}(t)=\tau_{\mathrm{a}}(\Theta, V, \Omega)-\tau_{\mathrm{g}}(\Omega),
$$

where $\Omega(t), J_{\mathrm{r}} \in \mathbb{R}$ denote the rotational speed and inertia of the rotor, respectively. The (quasi-steady) aerodynamic torque $\tau_{\mathrm{a}}(\Theta, V, \Omega): \mathbb{R} \times \mathbb{R} \times \mathbb{R} \rightarrow \mathbb{R}$ is a non-linear function of the blade-pitch angle $\Theta(t)$, wind speed $V(t)$ and rotor speed $\Omega(t)$, whilst the generator torque $\tau_{\mathrm{g}}(\Omega): \mathbb{R} \rightarrow \mathbb{R}$ in the above-rated wind conditions is defined as follows:

$$
\tau_{\mathrm{g}}(\Omega)=\frac{P_{\mathrm{sp}}}{n_{\mathrm{gb}} \Omega},
$$

where $P_{\mathrm{sp}} \in \mathbb{R}$ is the power set-point and $n_{\mathrm{gb}} \in \mathbb{R}$ is the gearbox ratio.

Next, a linear model is developed based on (1). In the above-rated wind conditions, for each operating wind speed $V^{*} \in \mathbb{R}$, there is corresponding equilibrium pitch angle 
$\Theta^{*}\left(V^{*}\right) \in \mathbb{R}$ and the rotor speed is operating at rated $\Omega^{*}$. The deviation notation is defined as follows:

$$
\omega(t)=\Omega(t)-\Omega^{*}, \theta(t)=\Theta(t)-\Theta^{*}, v(t)=V-V^{*} .
$$

The aerodynamic torque in (1) is linearised around the turbine operating conditions $\left(\Theta^{*}, V^{*}, \Omega^{*}\right)$ as follows:

$$
\begin{aligned}
& \tau_{\mathrm{a}}(\Theta, V, \Omega) \\
& \approx \tau_{\mathrm{a}}\left(\Theta^{*}, V^{*}, \Omega^{*}\right)+\frac{\partial \tau_{\mathrm{a}}}{\partial \theta} \theta(t)+\frac{\partial \tau_{\mathrm{a}}}{\partial v} v(t)+\frac{\partial \tau_{\mathrm{a}}}{\partial \omega} \omega(t) .
\end{aligned}
$$

Similarly, the generator torque in (1) is linearised as follows:

$$
\tau_{\mathrm{g}}(\Omega) \approx \tau_{\mathrm{g}}\left(\Omega^{*}\right)+\frac{\partial \tau_{\mathrm{g}}}{\partial \omega} \omega(t) .
$$

Substituting (2) into (1) yields the linear drive-train model as follows:

$$
J_{\mathrm{r}} \dot{\omega}(t)=\frac{\partial \tau_{\mathrm{a}}}{\partial \theta} \theta(t)+\frac{\partial \tau_{\mathrm{a}}}{\partial v} v(t)+\frac{\partial \tau_{\mathrm{a}}}{\partial \omega} \omega(t)-\frac{\partial \tau_{\mathrm{g}}}{\partial \omega} \omega(t) .
$$

For brevity, the linear drive-train model (3) is expressed in terms of the azimuth angle $\dot{\phi}(t)=\omega(t)$, defined as follows:

$$
J_{\mathrm{r}} \ddot{\phi}(t)+\left(\frac{\partial \tau_{\mathrm{g}}}{\partial \omega}-\frac{\partial \tau_{\mathrm{a}}}{\partial \omega}\right) \dot{\phi}(t)=\frac{\partial \tau_{\mathrm{a}}}{\partial \theta} \theta(t)+\frac{\partial \tau_{\mathrm{a}}}{\partial v} v(t) .
$$

Next, the nominal blade-pitch PI controller is presented. The linear model in (4) only considers the key drive-train dynamics whereas the torsion and other turbine dynamics to the drive-train are neglected. The rotor speed variations are mainly dominated at the low frequency that is typically lower than the rotational frequency of the blade ( $1 \mathrm{p}$ frequency). Thus, the controller design needs to avoid exciting other turbine dynamics. The typical blade-pitch PI control structure is as follows:

$$
\theta(t)=K_{\mathrm{p}} \omega(t)+K_{\mathrm{i}} \int_{0}^{t} \omega(\tau) d \tau=K_{\mathrm{p}} \dot{\phi}(t)+K_{\mathrm{i}} \phi(t),
$$

where $K_{\mathrm{p}}, K_{\mathrm{i}} \in \mathbb{R}$ denote the proportional and integral gains, respectively. Typically, the nominal blade-pitch control adopts the pole-placement method for choosing the $K_{\mathrm{p}}$ and $K_{\mathrm{i}}$ [8]. The pole-placement method is to assign the location of the eigenvalues of the closed-loop systems to achieve certain bandwidth and response. Substituting the controller (5) into the linear drive-train model (4) yields the closed-loop system mode, defined as follows:

$$
\begin{aligned}
J_{\mathrm{r}} \ddot{\phi}(t)+\left(\frac{\partial \tau_{\mathrm{g}}}{\partial \omega}-\frac{\partial \tau_{\mathrm{a}}}{\partial \omega}-K_{\mathrm{p}} \frac{\partial \tau_{\mathrm{a}}}{\partial \theta}\right) \dot{\phi}(t)- & K_{\mathrm{i}} \frac{\partial \tau_{\mathrm{a}}}{\partial \theta} \phi(t) \\
& =\frac{\partial \tau_{\mathrm{a}}}{\partial v} v(t) .
\end{aligned}
$$

Let the desired closed-loop eigenvalues denote as $\lambda_{\mathrm{cl}}=$ $-\zeta_{\Omega} \omega_{\Omega} \pm j \omega_{\Omega} \sqrt{1-\zeta_{\Omega}^{2}} \in \mathbb{C}$, where $\zeta_{\Omega}, \omega_{\Omega} \in \mathbb{R}$ are the damping ratio and natural frequency of the closed-loop system whilst $j:=\sqrt{-1}$. The desired eigenvalues ensure the frequency of the rotor speed regulation mode is sufficiently below the turbine tower frequency, where the typical values of the natural frequency $\omega_{\Omega}$ and damping ratio $\zeta_{\Omega}$ are $0.06 \mathrm{~Hz}$ and 0.7, respectively [9]. Based on (6), the $K_{\mathrm{p}}$ and $K_{\mathrm{i}}$ that

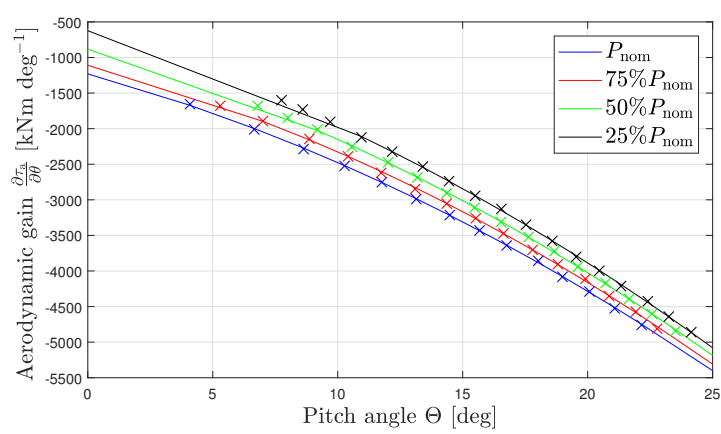

Fig. 1: Aerodynamic gains of the turbine in downregulation. The crosses and lines show the simulation data and their quadratic approximation.

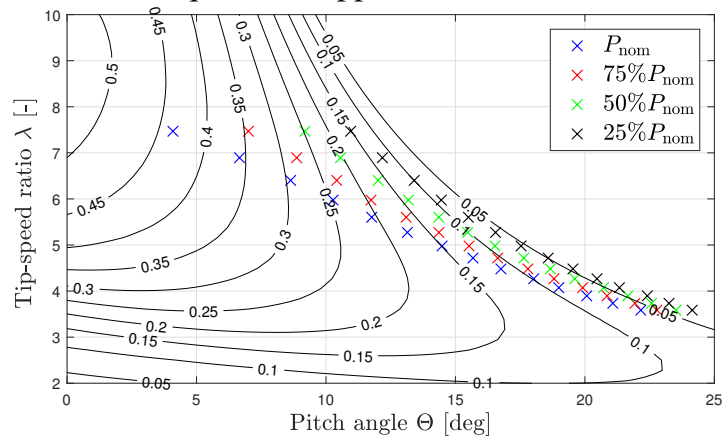

Fig. 2: The contour of the power coefficient $C_{\mathrm{p}}$ of the DTU 10MW turbine and different operating points in down-regulation.

gives the desired eigenvalues of the closed-loop system (6) are derived as follows:

$$
K_{\mathrm{p}}=\frac{2 \zeta_{\Omega} \omega_{\Omega} J_{\mathrm{r}}+\frac{\partial \tau_{\mathrm{a}}}{\partial \omega}-\frac{\partial \tau_{\mathrm{g}}}{\partial \omega}}{-\frac{\partial \tau_{\mathrm{a}}}{\partial \theta}}, K_{\mathrm{i}}=\frac{\omega_{\Omega}^{2} J_{\mathrm{r}}}{-\frac{\partial \tau_{\mathrm{a}}}{\partial \theta}},
$$

Notice that in the closed-loop model (6), the aerodynamic gain $\frac{\partial \tau_{\mathrm{a}}}{\partial \theta}$ varies dependent upon the operating conditions $\left(\Theta^{*}, V^{*}, \Omega^{*}\right)$. Thus, to ensure good closed-loop response, the blade-pitch controller typically adopts a gain-scheduling approach based on the operation conditions, where the aerodynamic gain $\frac{\partial \tau_{\mathrm{a}}}{\partial \theta}$ and aerodynamic damping $\frac{\partial \tau_{\mathrm{a}}}{\partial \omega}$ are formulated as a function of the operating pitch angle $\Theta^{*}$ and $\frac{\partial \tau_{g}}{\partial \omega}$ is a function of the operating rotor speed, defined as follows:

$$
\begin{aligned}
& \frac{\partial \tau_{\mathrm{a}}}{\partial \theta}=\left.\frac{\partial \tau_{\mathrm{a}}}{\partial \theta}\right|_{\Theta=0}\left(1+\frac{\Theta}{K_{1}^{\theta}}+\frac{\Theta^{2}}{K_{2}^{\theta}}\right) ; \\
& \frac{\partial \tau_{\mathrm{a}}}{\partial \omega}=\left.\frac{\partial \tau_{\mathrm{a}}}{\partial \omega}\right|_{\Theta=0}\left(1+\frac{\Theta}{K_{1}^{\omega}}+\frac{\Theta^{2}}{K_{2}^{\omega}}\right) ; \frac{\partial \tau_{\mathrm{g}}}{\partial \omega}=-\frac{P_{\mathrm{sp}}}{n_{\mathrm{gb}} \Omega^{* 2}},
\end{aligned}
$$

where $K_{1}^{\theta}, K_{2}^{\theta}, K_{1}^{\omega}, K_{2}^{\omega} \in \mathbb{R}$ are the coefficients of linear and quadratic terms in gain-scheduling. For example, aerodynamic gains in down-regulation are depicted in Figure 1. 
TABLE I: Parameters of the DTU 10MW reference wind turbine [10]

\begin{tabular}{|cc|cc|cc|} 
Nominal Power $P_{\text {nom }}$ & $10 \mathrm{MW}$ & Rotor orientation & Upwind & Rotor diameter & $178.3 \mathrm{~m}$ \\
Hub height & $119 \mathrm{~m}$ & Rated rotor speed & $9.6 \mathrm{rpm}(\approx 0.16 \mathrm{~Hz})$ & Rotational inertia of the rotor & $1.61 \times 10^{8} \mathrm{kgm}^{-2}$
\end{tabular}

\section{BLADE-PITCH CONTROLLER FOR DOWN-REGULATION: MOTIVATING EXAMPLE AND MAIN RESULT}

This section presents the problem of simply imposing the down-regulation strategy upon the existing nominal bladepitch controller and also the solution. Figure 2 depicts the power coefficient $C_{\mathrm{p}}$ surface for the DTU $10 \mathrm{MW}$ reference wind turbine [10], where the details of the turbines is listed in Table I. The points in Figure 2 are illustrated the operating points with respect to different power demand. It is clear in Figure 2, for down-regulation, the turbine operates at different tip-speed ratio $\lambda:=\frac{\Omega r}{V}$ and pitch angle $\Theta$ than nominal, where $r \in \mathbb{R}$ is the blade length. Based on the $C_{\mathrm{p}}$ surface and operating points in Figure 2, the aerodynamic gains $\frac{\partial \tau_{\mathrm{a}}}{\partial \theta}$ for different power demand are derived as shown in Figure 1. As for the following analysis, $75 \%$ of the nominal power $P_{\text {nom }}$ is chosen to represent the de-rating situations.

Lemma III.1. In nominal operations, the response and bandwidth of the closed-loop systems (6), formulated by the linear drive-train model (4) and the gain-scheduling controller (5) with the aerodynamic gains (8), are characterised by the desired eigenvalues $\lambda_{\mathrm{cl}}$.

Proof: It is trivail since the controller (5) is constructed based on the desired closed-loop eigenvalues $\lambda_{\mathrm{cl}}$, which characterises the response and bandwidth of the closed-loop system.

Notice the aerodynamic gain in the controller (5) is a quadratic approximation of the real aerodynamic gains. Thus, the natural frequency and damping ratio of the closed-loop system might vary slightly from the desired values 0.06 $\mathrm{Hz}$ and 0.7, respectively, as shown in Figure 3. Next, a motivating (sub-optimal) example is presented where the de-rating strategy is implemented upon the nominal pitch controller.

Lemma III.2. For down-regulation, the linear drive-train dynamics is defined as follows:

$$
J_{\mathrm{r}} \ddot{\phi}(t)+\left(\frac{\partial \tau_{\mathrm{g}, \mathrm{d}}}{\partial \omega}-\frac{\partial \tau_{\mathrm{a}, \mathrm{d}}}{\partial \omega}\right) \dot{\phi}(t)=\frac{\partial \tau_{\mathrm{a}, \mathrm{d}}}{\partial \theta} \theta(t)+\frac{\partial \tau_{\mathrm{a}, \mathrm{d}}}{\partial v} v(t) .
$$

where $\tau_{\mathrm{a}, \mathrm{d}}, \tau_{\mathrm{g}, \mathrm{d}}$ denote the aerodynamic and generator torques during de-rating. The response and bandwidth of the closed-loop system, formulated by the linear system (9) and the controller (5) based on the aerodynamic gains (8) are not characterised by the desired eigenvalues $\lambda_{\mathrm{cl}}$, resulting in excitation of other turbine dynamics.

Proof: Substituting the nominal controller (5) into (9) yields the (sub-optimal) closed-loop systems, where the natural frequency and damping ratio of such a closed-loop
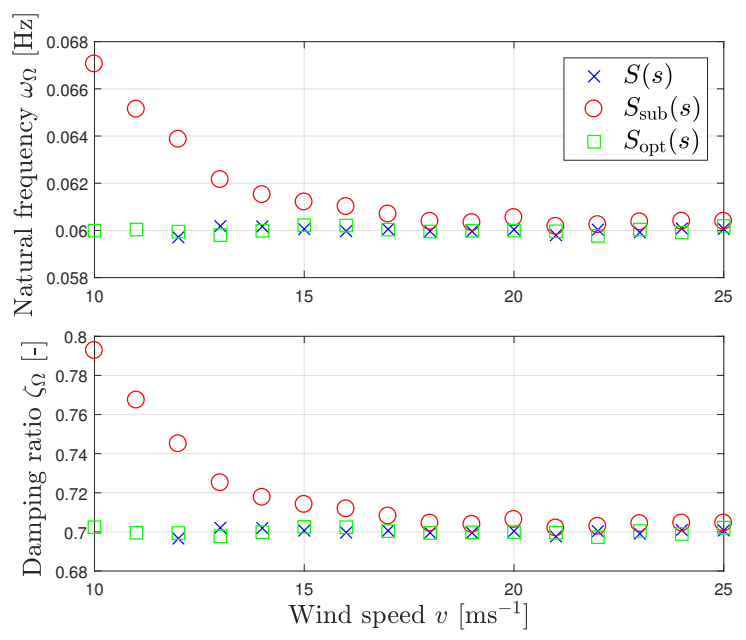

Fig. 3: The natural frequency and damping ratio of the closed-loop systems.

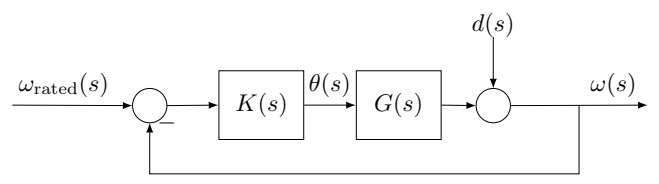

Fig. 4: Block diagrams of the closed-loop system.

system $\lambda_{\mathrm{cl}}$ are far from the desired position in the desired positions, as shown in Figure 3.

To investigate the effect of other turbine dynamics on the rotor speed control loop, transfer functions $G(s), G_{\mathrm{d}}(s) \in$ $\mathcal{R}$ are defined that maps the pitch angle $\theta$ to the rotor $\omega$ during nominal (4) and de-rating (9) operations, respectively, as follows:

$$
\begin{aligned}
& G(s):=\frac{\frac{\partial \tau_{\mathrm{a}}}{\partial \theta}}{J_{\mathrm{r}} s+\left(\frac{\partial \tau_{\mathrm{g}}}{\partial \omega}-\frac{\partial \tau_{\mathrm{a}}}{\partial \omega}\right)} \\
& G_{\mathrm{d}}(s):=\frac{\frac{\partial \tau_{\mathrm{a}, \mathrm{d}}}{\partial \theta}}{J_{\mathrm{r}} s+\left(\frac{\partial \tau_{\mathrm{g}, \mathrm{d}}}{\partial \omega}-\frac{\partial \tau_{\mathrm{a}, \mathrm{d}}}{\partial \omega}\right)} .
\end{aligned}
$$

The Laplace transform of the nominal controller (5) is defined as follows:

$$
K(s)=K_{\mathrm{p}}+K_{\mathrm{i}} \frac{1}{s} .
$$

Considering the closed-loop system as depicted in Figure 4, for the purpose of evaluating the effect of the disturbance $d(s)$, including the wind $v$ and other turbine dynamics to the rotor speed $\omega(s)$, the sensitivity functions are employed, defined as follows:

$$
S(s)=\frac{1}{1+G(s) K(s)}, S_{\mathrm{sub}}(s)=\frac{1}{1+G_{\mathrm{d}}(s) K(s)} .
$$




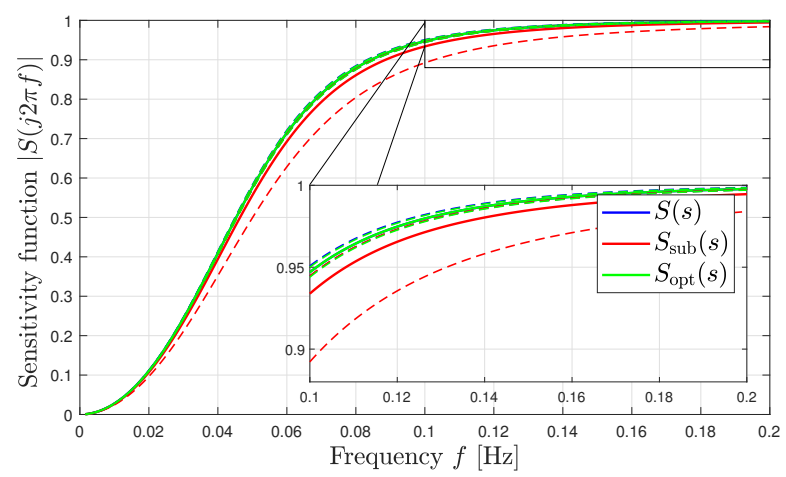

Fig. 5: The sensitivity functions. The dash lines show the minimum and maximum, whilst the mean is depicted by the solid line of different operating conditions.

Figure 5 shows the magnitude of the disturbance sensitivity functions $S(s), S_{\text {sub }}(s) \in \mathcal{R}$ for the nominal and sub-optimal de-rating closed-loop systems, respectively. The gain crossover frequency for $S_{\mathrm{sub}}(s)$ is higher and more uncertain than $S(s)$, that implies the controller (5) in down-regulation attenuates the disturbances or noises that it is not supposed to reduce, which could potentially excite other turbine dynamics.

Consequently, for the blade-pitch controller (5) not to excite other turbine dynamics and improve the closed-loop response, the sensitivity function and the eigenvalues (natural frequency and damping ratio) of the closed-loop system during down-regulation needs to be identical to those in nominal operations.

Theorem III.3 (Main result). Considering the nominal blade pitch controller (7) whose parameters $K_{\mathrm{p}}$ and $K_{\mathrm{i}}$ are updated based on the de-rated operations, defined as follows:

$$
\begin{aligned}
& K_{\mathrm{p}}=\frac{2 \zeta_{\Omega} \omega_{\Omega} J_{\mathrm{r}}+\frac{\partial \tau_{\mathrm{a}, \mathrm{d}}}{\partial \omega}-\frac{\partial \tau_{\mathrm{g}, \mathrm{d}}}{\partial \omega}}{-\frac{\partial \tau_{\mathrm{a}, \mathrm{d}}}{\partial \theta}}, K_{\mathrm{i}}=\frac{\omega_{\Omega}^{2} J_{\mathrm{r}}}{-\frac{\partial \tau_{\mathrm{a}, \mathrm{d}}}{\partial \theta}}, \\
& \frac{\partial \tau_{\mathrm{a}, \mathrm{d}}}{\partial \theta}=\left.\frac{\partial \tau_{\mathrm{a}, \mathrm{d}}}{\partial \theta}\right|_{\Theta=0}\left(1+\frac{\Theta}{K_{1, \mathrm{~d}}^{\theta}}+\frac{\Theta^{2}}{K_{2, \mathrm{~d}}^{\theta}}\right), \\
& \frac{\partial \tau_{\mathrm{a}, \mathrm{d}}}{\partial \omega}=\left.\frac{\partial \tau_{\mathrm{a}, \mathrm{d}}}{\partial \omega}\right|_{\Theta=0}\left(1+\frac{\Theta}{K_{1, \mathrm{~d}}^{\omega}}+\frac{\Theta^{2}}{K_{2, \mathrm{~d}}^{\omega}}\right), \\
& \frac{\partial \tau_{\mathrm{g}, \mathrm{d}}}{\partial \omega}=-\frac{P_{\mathrm{sp}}}{n_{\mathrm{gb}} \Omega *^{2}},
\end{aligned}
$$

then, the response and control bandwidth of the closedloop system during down-regulation, formulated from (9) and (13), are identical to the systems in nominal operations (6).

Proof: Substituting (13) into (9) yields the closed-loop system model during down-regulation:

$$
J_{\mathrm{r}}\left(\ddot{\phi}(t)+2 \zeta_{\Omega} \omega_{\Omega} \dot{\phi}(t)+\omega_{\Omega}^{2} \phi(t)\right)=\frac{\partial \tau_{\mathrm{a}, \mathrm{d}}}{\partial v} v(t),
$$

and the natural frequency and damping ratio of the closedloop system (14) are identical to those of the nominal closed- loop system (6), as shown in Figure 3. Moreover, considering the Laplace transform of (13) as $K_{\mathrm{d}}(s)$, combining the linear drive-train model during de-rating $G_{\mathrm{d}}(s)$ in (10) forms the sensitivity function $S_{\mathrm{opt}}(s):=\frac{1}{1+G_{\mathrm{d}}(s) K_{\mathrm{d}}(s)}$, which is nearly equivalent to the sensitivity function of the nominal closed-loop $S(s)$, that is illustrated in Figure 5 and such similarities can be confirmed by the $\nu$-gap metric [11]. The small difference between $S(s)$ and $S_{\text {opt }}(s)$ is a result of the quadratic approximation in aerodynamic characteristics in Figure 1.

\section{Numerical SimUlations}

\section{A. Simulation environment setting}

The turbine model used in this study is the DTU 10MW reference wind turbine [10] upon the HAWC2 platform [12]. This model includes many degrees-of-freedom such as the tower fore-after, side-to-side, in addition to the rotor and blade dynamics. The controller employed in this study is the open-source DTU basic controller ${ }^{1}$ [8] with down-regulation feature.

\section{B. Step wind case}

In these closed-loop simulations, two blade-pitch controllers in down-regulation were examined. The power setpoint was $75 \%$ of the nominal power. The baseline is the controller (5) based on the nominal aerodynamic gains (8) and it is compared with the proposed controller (13) with updates on the aerodynamic gains based on the power demand.

Figure 6 shows the sample time history of the rotor speed response, pitch angle and pitch rate subject to a step wind from $11 \mathrm{~ms}^{-1}$ to $12 \mathrm{~ms}^{-1}$. It is clearly shown that the pitch activities were better for the proposed controller, with a mere increase in the rotor speed variations, that fits the eigenvalue analysis in Section III and Figure 3.

\section{Turbulent wind cases}

The closed-loop simulations were conducted under a turbulent wind field with the mean speed of $16 \mathrm{~ms}^{-1}$ based on the IEC standard [13]. The histograms of the rotor speed and pitch rate variations are shown in Figure $7 \mathrm{a}$ and $7 \mathrm{~b}$. The proposed controller achieved reductions in pitch activities and slight increases in the rotor speed variations. The $1 \mathrm{~Hz}-$ equivalent fatigue loads on the key turbine components are summarised in Table II. Reductions on pitch activities resulted in better attenuation on the tower-base side-side loads, blade flap-wise, edge-wise loads and pitch bearing, with merely increases in the main shaft torsion and tower fore-aft loads.

\section{CONCLUSiON}

In this paper, the design of gain-scheduling blade-pitch controller for wind turbine down-regulation is presented. The proposed design retains the desired robustness, bandwidth and performance of the rotor speed regulation loop in both

\footnotetext{
${ }^{1}$ Available in GitLab: https://gitlab.windenergy.dtu.dk/OpenLAC/BasicDTUController
} 
TABLE II: Performance of the baseline and proposed blade-pitch control strategy in down-regulation

\begin{tabular}{c|cccccc} 
Controller & Main shaft torsion & Tower fore-aft & Tower side-side & Blade flap-wise & Blade edge-wise & blade-pitch bearing \\
\hline Baseline (5) [\%] & 100.00 & 100.00 & 100.00 & 100.00 & 100.00 & 100.00 \\
Proposed (13) [\%] & 100.21 & 100.43 & 98.53 & 98.23 & 97.34 & 97.71
\end{tabular}
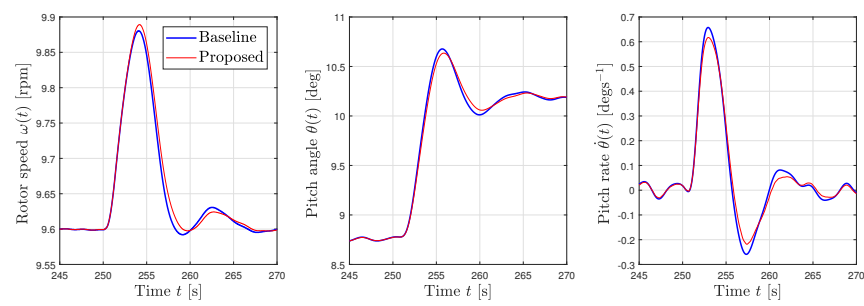

Fig. 6: The sample time histories of the baseline (blue) and proposed (red) controller in down-regulation.

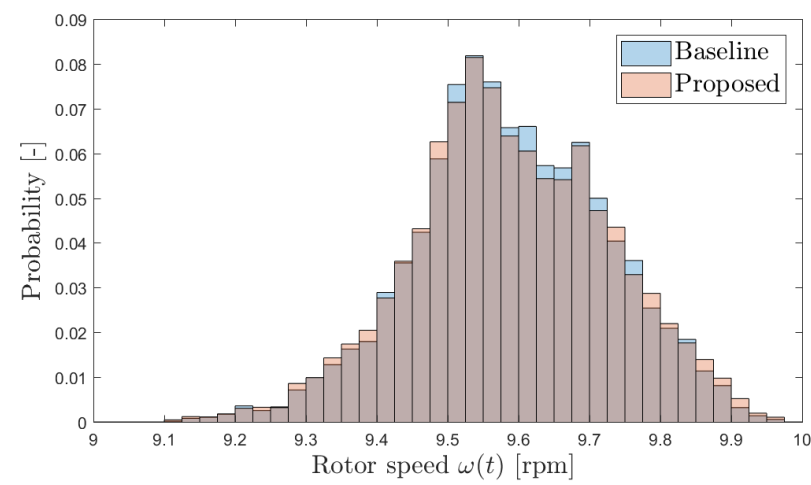

(a) The rotor speed.

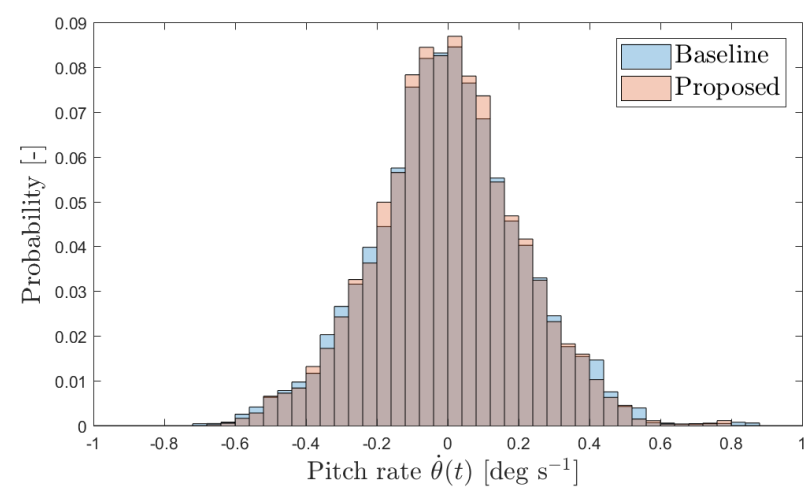

(b) The pitch rate.

Fig. 7: The histograms of the baseline (blue) and proposed (red) controller in down-regulation. nominal and de-rating operations. In the future, we look into the possibility of extending this blade-pitch control design to more down-regulation methods, for example, Const- $\lambda$ and Const- $\Omega$ in [3]. Also, the closed-loop eigenvalue analysis will be conducted with a higher degree-of-freedom drivetrain model.

\section{REFERENCES}

[1] M. Mirzaei, T. Gocmen, G. Giebel, P. E. Sorensen, and N. K. Poulsen, "Turbine Control strategies for wind farm power optimization," in 2015 American Control Conference (ACC), pp. 1709-1714, IEEE, jul 2015.

[2] C. Galinos, T. J. Larsen, and M. Mirzaei, "Impact on wind turbine loads from different down regulation control strategies," in DeepWind2018, 2018.

[3] M. Mirzaei, M. Soltani, N. K. Poulsen, and H. H. Niemann, "Model based active power control of a wind turbine," 2014 American Control Conference, no. July 2016, pp. 5037-5042, 2014.

[4] J. Zhu, K. Ma, M. Soltani, A. Hajizadeh, and Z. Chen, "Comparison of loads for wind turbine down-regulation strategies," 2017 11th Asian Control Conference (ASCC), pp. 2784-2789, 2017.

[5] W. H. Lio, M. Mirzaei, and G. C. Larsen, "On wind turbine downregulation control strategies and rotor speed set-point," The Science of Making Torque from Wind, 2018.

[6] M. H. Hansen, A. Hansen, T. J. Larsen, S. Oye, P. Sorensen, and P. Fuglsang, "Control design for a pitch-regulated, variable speed wind turbine," tech. rep., Ris $\varnothing$ Report, 2005.

[7] C. Tibaldi, L. C. Henriksen, M. H. Hansen, and C. Bak, "Effects of gain-scheduling methods in a classical wind turbine controller on wind turbine aeroservo-elastic modes and loads," 32nd ASME Wind Energy Symposium, no. January, pp. 1-12, 2014.

[8] M. H. Hansen and L. C. Henriksen, "Basic DTU Wind Energy controller," Tech. Rep. January, 2013.

[9] M. Hansen and F. Zahle, Aeroelastic Optimization of MW Wind Turbines, vol. 1803. 2011.

[10] C. Bak, F. Zahle, R. Bitsche, and T. Kim, "The DTU 10-MW reference wind turbine," in Danish Wind Power Research 2013, (Fredericia, Denmark), 2013.

[11] G. Vinnicombe, Uncertainty and Feedback. Imperial College Press, 2000.

[12] T. J. Larsen and A. M. Hansen, "How 2 HAWC2, the user's manual," tech. rep., 2007.

[13] IEC, "IEC 61400-1 Ed.3: Wind turbines - Part 1: Design requirements," vol. 2005, 2005. 